\title{
大气中持久性有机污染物被动采样技术及其在偏 远区域的应用研究进展
}

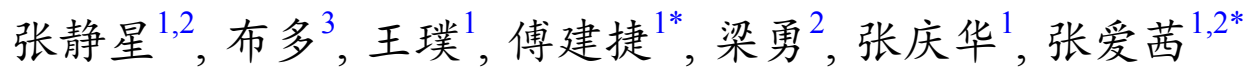

1. 中国科学院生态环境研究中心, 环境化学与生态毒理学国家重点实验室, 北京 100085

2. 江汉大学环境与健康研究院, 武汉 430056

3. 西藏大学理学院, 拉萨 850000

*通讯作者, E-mail: jjfu@rcees.ac.cn; aqzhang@rcees.ac.cn

收稿日期：2018-04-10; 接受日期：2018-05-17; 网络版发表日期：2018-09-20

中国科学院战略先导专项(编号: XDA2004050203)和国家自然科学基金(编号: 21677168, 21677116)资助项目

摘要持久性有机污染物(POPs)可通过“全球蒸馏效应”和“高山冷凝效应”等从污染源长距离传输至极地、高 山等偏远区域, 并在当地环境和生物体内不断富集放大, 给其脆弱的生态系统带来了风险. 被动式大气采样器 (PAS)被广泛应用于极地等偏远区域大气中POPs的大尺度采样, 为评价偏远地区大气中传统POPs的污染水平和 变化趋势，以及评估POPs履约成效做出了重要贡献。近年来，PAS还被应用于研究新型POPs在全球的迁移、转 化、归趋等环境行为, 取得了较好的效果. 本文介绍了常见的 5 种被动式大气采样技术(SPMD-PAS、PUF-PAS、 XAD-2-PAS、SIP-PAS和FTS)及其发展历程，并重点对其在南、北极和青藏高原等偏远区域的应用及这些区域 POPs污染现状进行了综述, 提出了该领域未来的发展趋势和需求.

关键词持久性有机污染物, 大气被动采样技术, 南极, 北极, 青藏高原

\section{1 引言}

1962年，Carson《俶静的春天》一书描述了人类 无节制使用农药后将面临一个没有鸟、蜜蜂和蝴蝶的 可怕世界，而书中所提及的化学污染物如滴滴涕 (DDTs)，至今仍是环境科学关注的热点. 在长期的研 究过程中, 科学家发现一些污染物在环境中具有持久 性、在生态系统中具有生物蓄积性、对生物具有一定 的毒性, 并且能长距离传输, 这类污染物被称为持久性 有机污染物(persistent organic pollutants, POPs). POPs
已在包括极地、高山、远洋、深海等全球各类生态系 统中检出 ${ }^{[1 \sim 4]}$, 对全球生态安全及人类健康造成了风 险 ${ }^{[5 \sim 7]}$. 2001年联合国环境规划署(United Nations Environment Programme, UNEP)通过了消除或限制POPs 生产、使用及排放的《关于持久性有机污染物的斯德 哥尔摩公约》(简称公约), 公约于 2004年生效, 第一批 列入了12种(类)化学污染物, 截至2017年年底, 已有 28 类化合物被列入POPs名单, 3 类化合物在审查中 ${ }^{[8]}$.

POPs具有一定的挥发性, 大气是其扩散和长距离 传输的重要媒介, 因此大气中POPs的赋存水平是其环

引用格式: Zhang J, Bu D, Wang P, Fu J, Liang Y, Zhang Q, Zhang A. Progress on the passive air sampling techniques and occurrence of persistent organic pollutants in remote areas. Sci Sin Chim, 2018, 48, doi: 10.1360/N032018-00089 
境行为研究的基础, UNEP全球POPs监测计划也将大 气作为监测对象来评估公约效力 ${ }^{[9]}$. 大气中POPs一般 在 $10^{-5} \sim 10^{1} \mathrm{ng} / \mathrm{m}^{3}$ 水平, 而结合最终进样量, 分析仪器 的绝对检出限一般 $>10 \mathrm{fg}^{[10 \sim 12]}$, 因此采样体积至少需 在 $1 \mathrm{~m}^{3}$ 以上，对大气样品的直接分析受到了极大的限 制. 实际监测中一般先对大气中POPs富集后再进行前 处理和仪器分析. 根据采样动力的不同, 大气采样器分 为主动和被动两类 ${ }^{[13]}$. 主动式大气采样器 (active air samplers, AAS)通过采样洜加流量计精确控制大气采 样量, 通过滤膜和吸附介质可分别采集大气颗粒相和 气相中的POPs, 是目前采集大气中POPs最理想的装 置. 但AAS设备体积大、价格昂贵, 采样时需电力供 应和专业人员维护, 且只能通过增加采样频率来表征 较长时间段大气中POPs平均水平, 因此不适合野外、 偏远地区及大区域范围内的同步采样. 被动式大气采 样器(passive air samplers, PAS)采用对目标化合物具 有较高分配系数的吸附材料, 依赖化合物自身的扩散 作用被吸附材料捕获, 实现样品采集, 采样完成后通 过分析吸附材料中POPs的含量来表征采样期间大气 中POPs赋存水平. 与 $\mathrm{AAS}$ 相比, PAS采样周期较长、造 价低廉、体积小携带方便、采样期间无需电力供应和 特别维护, 非常适合于大尺度采样. 自2002年起, PAS 便被应用于综合大气沉降网络(Integrated Atmospheric Deposition Network, IADN $)^{[14]}$ 、全球大气被动采样监 测网络(Global Atmospheric Passive Sampling, GAPS) ${ }^{[15]}$ 以及欧洲监测与评估计划(European Monitoring and Evaluation Programme, EMEP) ${ }^{[16]}$ 等大尺度大气 监测项目中. 然而, PAS采样速率受采样材料、气象条 件等众多因素的影响, 且不能提供准确的采样体积, 因 此也限制了其更加广泛的应用.

对南极、北极和青藏高原等偏远区域大气中 POPs的研究 ${ }^{[17]}$, 一方面有助于研究POPs在大尺度下的 迁移、转化与归趋等环境行为, 另一方面偏远区域作 为全球大气环境的背景区域, 对这些地区大气中POPs 赋存的持续研究可以了解全球人类生存环境的变化, 对评估POPs给偏远区域生态系统可能造成的风险和 全球POPs削减、公约的履约进程具有重要的意义. 本 文以大气中POPs被动采样技术发展为主线, 阐述了 PAS的应用前景, 并对南极、北极和青藏高原等偏远 区域大气POPs污染现状进行概述, 提出未来大气POPs 被动采样需关注的问题, 并且对极地等偏远区域POPs
研究进行了展望.

\section{2 大气POPs被动采样技术}

在PAS使用前, 已有研究通过松针、树皮、苔藓 及地衣等生物介质中污染物的浓度来反映大气中污染 物水平的变化 ${ }^{[18]}$, 甚至有研究将洁净区域采集的苔藓 放置到特定区域来采集污染物 ${ }^{[19]}$. 生物介质一般具有 蜡脂层表面或高表面积结构, 能有效地吸附大气中的 有机污染物, 因此被广泛地用于反映大气中污染物的 水平, 即生物被动采样. 然而, 通过生物表征大气中污 染物的浓度和污染特征并不稳定 ${ }^{[18,20]}$, 一方面生物生 长时间、生长阶段差异都会影响其对POPs的吸附, 另 一方面, 生物种类的空间分布具有局限性, 不同种类的 生物有可能对污染物具有选择性富集, 因此很难通过 一种生物指示物在大尺度上对污染物的浓度水平及特 征进行横向比较. 基于对生物吸附的认识, 引入均一特 性的吸附剂进而构建的PAS装置则应运而生. PAS可以 避免生物采样法中生物体本身差异带来的不确定性, 将同种吸附剂的PAS应用到大尺度的研究中, 能更好 地对POPs等污染物的环境行为进行深入研究.

PAS的采样原理主要基于Fick第一定律和Whit$\operatorname{man}$ 双膜理论, 采样期理论上分 3 个阶段 ${ }^{[21]}$ —线性吸 附、曲线吸附和平衡期(图1). 被动采样介质(PSM)上 吸附的目标物的质量 $m(\mathrm{ng})$, 除以这段时间采集的大 气体积 $V_{\text {air }}\left(\mathrm{m}^{3}\right)$, 即为采样时期目标物在大气中的平均 浓度 $C_{\text {air }}\left(\mathrm{ng} / \mathrm{m}^{3}\right.$ ). PSM的 $R$ 值(采样速率 $\left(\mathrm{m}^{3} / \mathrm{d}\right.$ )) 直接关系 到采样体积 $\left(V_{\mathrm{air}}=R t\right)$ 的准确性, 因此只有处于线性吸附 阶段的PAS才能对一段时间内大气中的污染物水平进 行较为准确地定量. 在实际操作中, $R$ 主要通过与 $\mathrm{AAS}$ 对比, 逸失参考化合物(depuration compounds, DCs/ performance reference compounds, PRCs) ${ }^{[22 \sim 26]}$ 和六氯 苯(HCB)类化合物反推法 ${ }^{[27,28]}$ 进行计算及校正. 根据 $\mathrm{PSM}$ 不同, PAS可分为半渗透膜采样器(SPMD-PAS)、 聚氨酯泡沫盘采样器(PUF-PAS)、浸渍XAD-4粉末的 PUF盘采样器(SIP-PAS)以及苯乙烯-二乙烯基苯共聚 物树脂采样器(XAD-2-PAS). 采样器装置主要包括半 封闭式采样器外罩和吸附介质, 外罩的形状随吸附介 质的不同而设定. 关于SPMD-PAS、PUF-PAS等采样 器装置、采样原理及采样速率的计算, 国内外均有文 献对其详细地介绍 ${ }^{[15,29 ~ 33]}$. 


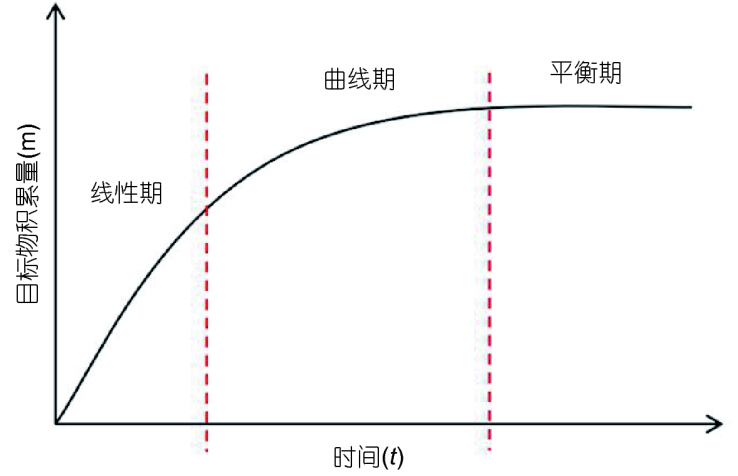

图 1 PAS采样三阶段——线性期、曲线期、平衡期 ${ }^{[21]}$ (网 络版彩图)

Figure 1 The three stages of PAS sampling: linear, curve, and equilibrium adsorption [21] (color online).

\section{1 半渗透膜采样器}

SPMD采样器最早被用来监测水环境中的亲脂性 有机物 ${ }^{[34,35]}$, 直到1993年, Petty 等 ${ }^{[36]}$ 才将内置三油酸甘 油酯的SPMD-PAS应用于空气中有机污染物的采样, 并通过与 $\mathrm{AAS}$ 数据对比, 计算出 SPMD对PCBs的采样 速率 $R$ 为 $2.17 \mathrm{~m}^{3} / \mathrm{d}$. SPMD采样器虽受风速影响不大, 但温度会对其造成一定影响. Ockenden等 ${ }^{[37]}$ 将SPMDPAS用于城郊结合地区采样, 通过大流量主动采样器 (HiV-AAS) 校正, 得到夏、冬季SPMD-PAS的 $R$ 值差异 较大, 分别为 1.9 和 $7.6 \mathrm{~m}^{3} / \mathrm{d}$, 经过分析认为 $R$ 值差异较 大的主要原因是低温下大气中有机污染物以更快的速 度吸附到已渗透在聚乙烯膜外的脂质层中, 膜内外较 大的浓度梯度使得污染物跨膜驱动力增加, 因此 $R$ 值 冬季较夏季高. Ockenden等将SPMD-PAS应用于英国 南部 $\left(50^{\circ} \mathrm{N}\right)$ 至挪威北部 $\left(70^{\circ} \mathrm{N}\right)$ 的纬度横断面的 10 个位 点的大气PCBs监测, 结果表明, 大气中低氯代PCBs单 体(如tetra-CBs)在总PCBs中浓度占比随纬度上升而增 加, 高氯代PCBs单体则呈相反的趋势, 这是由于高氯 代PCBs单体相对于低氯代单体而言，挥发性较弱，而 纬度的升高往往伴随着温度的下降, 使得高氯代PCBs 在较低纬度被冷凝捕集，该研究也证实了 PCBs在随大 气长距离迁移过程中的分馏现象 ${ }^{[38]}$. Van Drooge 等 ${ }^{[22]}$ 将S P MD - PAS 应用于比利牛斯山不同海拔高度 (1600、2240和 $2600 \mathrm{~m}$ )采样, 通过DCs的损失率计算了 $R$ 值, SPMD-PAS监测的大气PCBs浓度与HiV-AAS结 果具有较好的一致性.
SPMD-PAS采样容量大，吸附线性区间可以长达 数年, 并且造价低廉、结构简单, 实验数据与 $\mathrm{AAS}$ 数 据一致性较好，但聚乙烯薄膜及亲脂性采样介质决定 了其一方面在采集大分子污染物时存在扩散障碍，另 一方面不能有效吸附疏油性污染物, 如长链FTOHs ${ }^{[39]}$. 此外, SPMD-PAS采样期间三油酸甘油酯会渗透到薄 膜外层并黏附部分大气颗粒物, 因此样品前处理过程 包括膜外层颗粒物及脂质去除或膜外层目标物振荡提 取、膜内目标物提取、凝胶渗透色谱(GPC)除脂以及 复合填充柱净化等步骤，操作过程繁琐，容易引入污 染, 并且聚乙烯薄膜和三油酸甘油酯均可吸附目标物 质, 采样动力学过程比较复杂. dos Reis等 ${ }^{[40]}$ 通过将 $\mathrm{XAD}$ 树脂替代三油酸甘油酯作为SPMD-PAS中的填充 材料, 并将改进后的采样器应用于室内空气中多环芳 烃(PAHs)的监测, 获得了较好的效果.

\section{2 聚氨酯泡沫盘被动采样器}

PUF早期被用于水体中金属衍生物及有机污染物 的浓缩和萃取 ${ }^{[41,42]}$. 1974年, Bidleman等 ${ }^{[43]}$ 将PUF作为 吸附介质用于大体积大气采样装置采集大气气相中的 PCBs. 2000年, Shoeib和Harner ${ }^{[21]}$ 将PUF放置在网架上 采集室内空气中的PCBs、多氯荎(PCNs), 发现PUF单 位面积大气采样速率与SPMD一致, 对一些低分子量 化合物而言，其在PUF上的线性吸附时间长于SPMD, 如PCB-18在SPMD和PUF上达到吸附平衡的时间分别 为 50 和 $140 \mathrm{~d}$, 证明了 PUF具有应用于PAS的潜力. 随后, Harner等 ${ }^{[44 ~ 46]}$ 设计出“双碟型”外罩的PUF-PAS，并将 这种采样器应用于欧洲 22 个国家的城市、乡村及偏远 地区大气中PCBs、多溴二苯醚(PBDEs)、PAHs、 PCNs和有机氯农药(OCPs)等的监测, 发现PUF-PAS获 取的大气污染物浓度数据与 $\mathrm{AAS}$ 结果有很好的一致 性. PUF-PAS相对于SPMD-PAS有较大优势: 采样机理 相对明确、采样介质安装简单, 不容易引入污染 ${ }^{[47]}$, 同时, PUF盘的萃取可以通过传统的索氏提取和加速 溶剂萃取进行, 前处理相对简单. PUF-PAS能有效地 监测POPs点源排放, 反映不同功能区区域POPs的空间 分布 ${ }^{[25,46,47]}$, 也可以用于阐述POPs土-气交换行为 等 ${ }^{[48]}$.

但PUF-PAS也存在明显的不足，一般情况下PUFPAS“双碟型”外罩能有效减弱风速对采样速率的干扰, 其采样速率 $R$ 为 $4.5 \mathrm{~m}^{3} / \mathrm{d}$ 左右, 然而, 当外部风速较高 
( $>3.74 \mathrm{~m} / \mathrm{s})$ 时, PUF-PAS内部气流速率增大, 采样速率 也会随之增加, 风速约为 $7 \mathrm{~m} / \mathrm{s}$ 时, PUF-PAS的 $R$ 值急剧 增至 $40 \mathrm{~m}^{3} / \mathrm{d}^{[49]}$. Li等 ${ }^{[50]}$ 将PUF-PAS根据一定的高度 梯度安装在气象塔上, 发现在高、低位点 $R$ 差值较大 一离地面 $15 \mathrm{~m}$ 处 $R_{15} \mathrm{~m}$ 为 $7.0 \mathrm{~m}^{3} / \mathrm{d}$, 而离地面 $320 \mathrm{~m}$ 处 $R_{320 \mathrm{~m}}$ 达到 $11 \mathrm{~m}^{3} / \mathrm{d}$, 进一步对气象塔不同高度位点处的 风速与对应的PUF-PAS的 $R$ 值回归分析, 发现二者间存 在指数相关. 已知采样点平均风速的情况下, 可以通过 风速与对应的 $R$ 值的回归方程, 提高 $R$ 值的准确性 ${ }^{[51]}$, 但不同风速下 $R$ 值极大的波动也限制了 PUF-PAS在极 地等偏远区域的应用. 极地、高山等偏远区域气象条 件复杂, 不同位点风速差异极大, 通过平均风速得到 $R$ 值计算污染水平仍会导致较大的误差. 此外, PUF-PAS 装置由于其本身体积较大, 在高风速下受到的外力也 较大, 采样过程中很可能出现装置受损的情形(图2). 同时, 研究表明PUF-PAS对污染物的吸附性能也存在 不足, 其线性采样期一般小于 3 个月 ${ }^{[52,53]}$, 对分子量较 小(挥发性较强)的POPs, 易发生吸附饱和, 因此较适合 监测大气POPs季节性变化, 不适宜用于极地等采样频 率需求为一年一次的偏远区域长期采样 ${ }^{[54]}$. GAPS中 PUF-PAS采集的大气样品中约 $80 \%$ 位点的氟调醇 (FTOHs)均低于检出限 ${ }^{[54]}$, 而同时期XAD-PAS等其他 大气采样器的研究发现, FTOHs广泛存在于大气中, 其浓度在 $1 \sim 10^{2} \mathrm{pg} / \mathrm{m}^{3}$ 左右 ${ }^{[54,55]}$, 这可能是因为FTOHs 挥发性较强, PUF对其吸附性能较弱, 有效采样体积仅 为 $0.94 \mathrm{~m}^{3}$, 仅在数小时内 PUF对FTOHs的吸附即达到 平衡, 这说明通过PUF-PAS对新型POPs采样之前需要 进行必要的评价.

总体而言, PUF-PAS设计简单、安装方便、成本

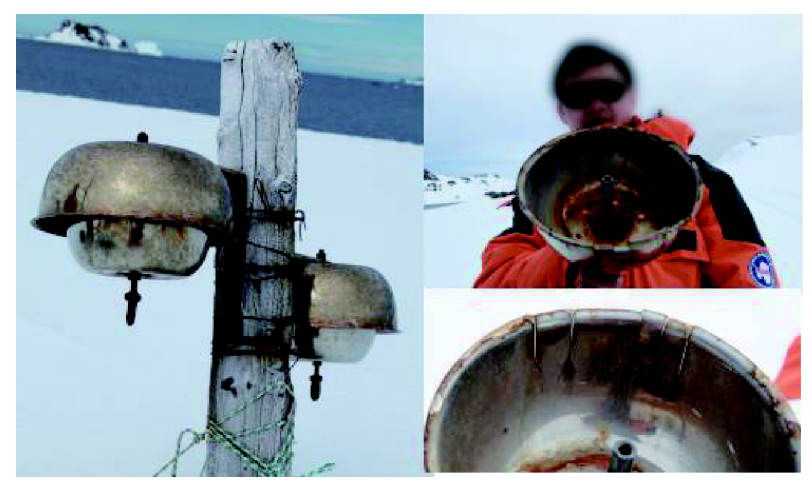

图 2 PUF-PAS应用于南极地区大气采样(网络版彩图) Figure 2 The application of PUF-PAS in Antarctica (color online).
低廉, 是UNEP推荐的大规模大气监测采样装置 ${ }^{[56]}$, 已 被用于全球大气中多种POPs的大尺度研究 ${ }^{[14,45]}$.

\section{3 高分子树脂聚合物被动采样装置}

2003 年, Wania等 ${ }^{[57]}$ 通过将XAD-2 填充于直径 $2 \mathrm{~cm}$ 、长 $20 \mathrm{~cm}$ 的不锈钢网制作的圆柱形容器内, 并将 其悬挂于圆柱型不锈钢罩筒内, 成功设计了XAD-2PAS, 并通过XAD-2-PAS对五大湖区域以及加拿大北 极Alert等地区大气中的OCPs进行了监测. 与PUF-PAS 等被动采样器相比, XAD-2-PAS吸附容量大、吸附性 能不受相对湿度影响且其线性吸附时间长于 1 年 ${ }^{[58 \sim 60]}$. 其优异的吸附性能可同时采集理化性质多样化的 POPs, 强大的吸附容量既能用于包括强挥发性POPs 在 内的月份/季节性监测, 又适用于偏远区域大气POPs长 期监测, 且可避免PUF-PAS 可能发生的吸附饱和, 很好 地满足了对大气POPs的时空监测 ${ }^{[57]}$.

众多学者对XAD-2-PAS的采样机理、采样动力 学影响因素及采样速率 $R$ 值等方面进行了系统研 究 $^{[27,57 \text { 67] }}$, 目前XAD-2-PAS已成为包括偏远区域在内 的全球范围大气POPs监测的有效工具. XAD-2-PAS采 样速率的主要影响因素有温度、大气压力、风速以及 目标物的理化性质等 ${ }^{[59,60,68]}$. Wania等 ${ }^{[57]}$ 将XAD-2-PAS 采样介质替换为硅胶并进行了风洞实验, 结果表明, 在 外部5 15 m/s 风速范围内, 硅胶对空气中的水分子吸 附速率基本稳定, 因此认为在该风速范围内, XAD-2PAS对大气中有机污染物也能够维持稳定的采样速率. 他们通过在加拿大北极Alert地区设置一系列不同采 样周期的XAD-2-PAS采集大气中的OCPs, 最终估算出 $\mathrm{XAD}-2-\mathrm{PAS}$ 的 $R$ 值为 $0.52 \mathrm{~m}^{3} / \mathrm{d}^{[57]}$. 该 $R$ 值在后续一些缺 乏AAS校正的研究中得到了广泛的应用 ${ }^{[69-72]}$. 2005年, Gouin等 ${ }^{[60]}$ 将XAD-2-PAS、PUF-PAS、HiV-AAS 3种 采样器用于热带地区(Antonio de Belen), 该研究得到 $\mathrm{XAD}-2-\mathrm{PAS}$ 的 $R$ 值为 $2.1 \mathrm{~m}^{3} / \mathrm{d}$, 说明采样环境对XAD-2$\mathrm{PAS}$ 的 $R$ 值有较大影响, 因此环境条件差异较大的位点 间, $R$ 值应合理评估.

Wang 等 ${ }^{[73]}$ 归纳已有研究中XAD-2-PAS 的 $R$ 值与 当地采样点的 $T 、 P$ 数据, 总结出 $R$ 值 $\left(\mathrm{PSR}_{\mathrm{WL}}\right)$ 的经验公 式(式(1)), 对青藏高原不同位点大气中POPs进行半定 量研究 ( $T$ 为绝对温度, $\mathrm{K} ; P$ 为大气压力, $\mathrm{hPa}$ ):

$$
\mathrm{PSR}_{\mathrm{WL}}=0.16 T^{1.75} / P-2.14\left(r^{2}=0.92\right),
$$


但是后续研究表明, 在青藏高原, 低风速地区鲁朗、拉 萨 (平均风速分别为 1 和 $1.6 \mathrm{~m} / \mathrm{s}$ ), $\mathrm{AAS}$ 校正获得的 XAD-2-PAS的 $R$ 值与 $T^{1.75} / P$ 存在正相关关系, 而当包含 较高风速地区 (纳木错, 平均风速为 $3.3 \mathrm{~m} / \mathrm{s}$ ) 在内时, 相 关性则较差. 这说明风速对XAD-2-PAS采样速率的影 响需要进一步评估, Gong等 ${ }^{[67]}$ 结合风速 $(v, \mathrm{~m} / \mathrm{s})$ 以及 $\mathrm{AAS}$ 校正法对 $R$ 值 $\left(\mathrm{PSR}_{\mathrm{m}}\right)$ 的计算进行了优化(式(2)):

$$
\mathrm{PSR}_{\mathrm{m}}=\left(0.16 T^{1.75} / P-2.07\right) \mathrm{e}^{0.92 v-2.12},
$$

经验证明该公式的准确性和通用性较好, 有助于气象 条件差异较大的采样位点XAD-2-PAS数据间的比较.

有研究发现, HCB在大气中分布较为均匀, 通过大 气中 $\mathrm{HCB}$ 平均浓度来计算不同采样位点特异性的 $R$ 值, 也可以达到抵消气象条件差异的效果, 获得的大气 POPs数据间可进行较好的比较 $[27,28]$.

XAD-2-PAS采样机理方面, Zhang等 ${ }^{[61,62]}$ 通过一系 列研究, 否定了传统PAS采样机理“Whitman双膜理 论, ${ }^{[21]}$ 中化合物在PSM内部分布均匀且PSM侧传质阻 力可忽略的观点, 提出新的PAS采样原理“三过程吸附 假设”, 认为目标物从空气相到PSM侧的传质过程中依 次有3个阶段: (1) 目标物从空气介质扩散至PSM表面, 并被吸附; (2) 目标物在PSM内部孔隙空气间扩散传 质; (3) 目标物在PSM内部孔隙固相材料与孔隙空气 相之间进行吸附和解吸. 这一假设支持了Chaemfa 等 ${ }^{[52]}$ 提出的“两阶段吸附机制”—PUF-PAS采样前期 (包含图1中的线性期), 目标物在PUF上的吸附存在“先 快后慢”的现象, 首先目标物以较快的速度吸附到PSM 表面, 然后进入到PSM内部, 因为存在传质阻力, 而导 致其在PSM内部传质速度较慢. 具体PAS“三过程吸附 假设”相关内容, 国内外已有研究学者对其做了详细阐 述 $^{[62,68,74]}$.

\subsection{XAD-4树脂浸渍的PUF盘被动采样器}

针对PUF-PAS在采集大气中一些强挥发性、极性 POPs时线性期较短的问题, Shoeib 等 ${ }^{[75]}$ 利用XAD树脂 吸附容量大、吸附化合物种类丰富等特点, 将XAD-4 粉末浸渍到PUF盘上, 设计出了SIP-PAS. SIP-PAS与 PUF-PAS相似, 能有效采集大气中传统POPs (PCBs、 OCPs等), 而且对强挥发性、新型POPs以及类POPs污 染物(FTOHs、挥发性甲基硅氧烷(VMSs)等)的吸附容 量更大, 线性吸附期更长, 是PUF-PAS很好的补充或替
代工具, 已被应用于 GAPS 等大尺度研究计划 中 $^{[26,54,75 \sim 80]}$.

SIP-PAS的 $R$ 值可通过AAS校正法 ${ }^{[75,79]}$ ，直接引用 PUF-PAS采样速率法 ${ }^{[54]}$ 、DCs 校正法 ${ }^{[26,77]}$ 得到. 不同 研究中AAS校正法估算的SIP-PAS的 $R$ 值 $\left(\mathrm{m}^{3} / \mathrm{d}\right)$ 差异较 大, 通常在0.6 6.2 $\mathrm{m}^{3} / \mathrm{d}$ 之间 ${ }^{[75,78 \sim 80]}$. SIP-PAS与PUFPAS的PUF盘几何尺寸相同，因此PUF-PAS、SIP-PAS 同时进行采样时, 通过DCs法获得PUF-PAS的 $R$ 值, 也 可同时应用于SIP-PAS ${ }^{[54]}$. 也有研究用DCs法分别校正 SIP-PAS和PUF-PAS的 $R$ 值, Liu等 ${ }^{[26]}$ 用SIP-PAS、PUFPAS分别采集北京大气中的有机磷酸脂(OPEs), 以 $\mathrm{D}_{15^{-}}$ 磷酸三苯酯 $\left(\mathrm{D}_{15}-\mathrm{TphP}\right)$ 为 $\mathrm{DC}$, 最后得到两种采样器的 $R$ 值分别为 1.7 和 $1.5 \mathrm{~m}^{3} / \mathrm{d}$. Ahrens 等 ${ }^{[77]}$ 在全氟化合物 (PFASs) 采样的研究中, 同时通过AAS与DCs法对SIPPAS的 $R$ 值进行了计算, 两者存在很好的一致性. SIPPAS对目标物的采集由PUF盘和XAD两者共同完成, 因此SIP-PAS吸附机制还需要进一步明确 ${ }^{[75]}$.

与PUF-PAS相比, SIP-PAS制备过程相对繁琐, 目 标物为PBDEs等广泛添加于室内物品的POPs及类 POPs时, 实验室空白的控制是一大挑战 ${ }^{[76]}$. 此外, SIP 盘浸渍的XAD-4量存在一定偏差 $(\sim 15 \%)^{[75]}$, 采样过程 中SIP盘上的XAD-4有可能产生损失, 会对该采样器的 平行性以及所获数据的可比性产生影响.

\section{5 “流入式”采样器}

上述PAS对目标物的采集均通过采样外罩减弱空 气扰动, 大气中污染物扩散到PSM表面并被吸附, 速度 较慢, 正常情况下采样速率在 $10^{0} \sim 10^{1} \mathrm{~m}^{3} / \mathrm{d}^{[37,52,54,60]}$, 需 要较长的采样周期(偏远地区采样周期一般需数月到 1 年)才能保证吸附的污染物被仪器检出, 时间分辨率受 到限制. Xiao 等 ${ }^{[81]}$ 于 2007 年设计了一种可随风向实时 调整采样的“流入式”采样器(flow-through sampler, FTS), FTS由安装在滚珠轴承柱子上的横向不锈钢空 气流通管、采样器顶部、流通管出口处的风速计以及 微电池数据记录仪等组成. FTS 主要依靠风力对其流 通管叶轮型尾部的作用力来随时调整流通管头部朝 向, 使得大量气流流经流通管内部, 同时气流中的目标 物被流通管中间部位的采样介质(多层PUF盘)截留, 随 后气流流向流通管末端, 并从出口处排出. FTS根据风 速计和记录仪在采样期间记录的风速数据确定出大气 样品体积 $V_{\mathrm{air}}$; 通过大气样品体积 $V_{\mathrm{air}}$ 与采样介质上积 
累的目标物的量 $m$, 最终推算出大气中目标物浓度 $C$ $\left(C=m / V_{\text {air }}\right)$. Xiao 等 ${ }^{[81]}$ 设计的FTS 的 $R$ 值可轻松达到 $15 \mathrm{~m}^{3} / \mathrm{d}$, 在风季甚至能达到 $100 \mathrm{~m}^{3} / \mathrm{d}$, 采样能力几乎与 $\mathrm{AAS}$ 平齐, 大大提高了采样的时间分辨率. 根据需要, FTS 的采样周期在 1 天到 1 个月不等, 采样结果与HiVAAS有很好的一致性, 并且具有较好的重现性 ${ }^{[81 ~ 83]}$. 但FTS 也存在一些不足, 外界风速过高或过低 (1 10 m/s范围外)时, 采集空气样品有一定困难; 而且 恶劣环境(暴风雨天气)下, 也存在FTS损坏和丢失风 险 ${ }^{[84]}$. 此外, FTS的制作成本也较高.

\section{PAS在偏远区域的应用}

偏远区域一般缺乏电力等基础设施, 通过AAS在 大尺度上对多个采样点同时进行采样比较困难, PAS 可评估一段时间内大气中POPs平均污染水平和污染 特征, 因此PAS被广泛地应用于偏远地区采样, 成为研 究偏远区域大气中POPs源、汇等环境行为的有效 手段.

与人类密集生活区相比，极地等偏远区域一般具 有风速大、地形复杂、气象条件变化大且记录信息少 等特点, 而风速和地形等因素都有可能影响PAS的 $R$ 值, 因此准确合理的 $R$ 值对 PAS 表征大气POPs的真实 水平尤为重要. 例如, XAD-2-PAS的 $R$ 值在乡村、城市 等常规区域位点对风速、风角度不敏感, 但是在特殊 地形下XAD-2-PAS的采样速率随风速波动极大. 中国 科学院青藏高原研究所王小萍团队 ${ }^{[67]}$ 在青藏高原的 研究中发现, 在采样器下方有斜坡的条件下, 风速为 $4.4 \mathrm{~m} / \mathrm{s}$ 时, $R$ 值可高达 $40 \mathrm{~m}^{3} / \mathrm{d}$. 为减弱地势和风速等对 $R$ 值的影响, 他们在XAD-2-PAS圆柱型采样筒底部添 加4层重叠且间隔 $1 \mathrm{~cm}$ 的整流板(图3), 流体力学模拟 及采样器内部气流速度测定均表明, 整流板的存在有 效地减弱了外部气场对采样器内部气流的扰动, $R$ 值 波动较小. 经改进的XAD-2-PAS用于现场试验后发现, 在各种地形和风速下 $R$ 值均比较稳定. 此外, 在高风速 环境中采样时的 $R$ 值也可以根据适用于微风环境下的 采样速率——温度/大气压力的经验公式(式(1))来推 算. 使用这种改进后的XAD-2-PAS可增强偏远区域不 同地势、气象条件下大气POPs监测数据间的可对比

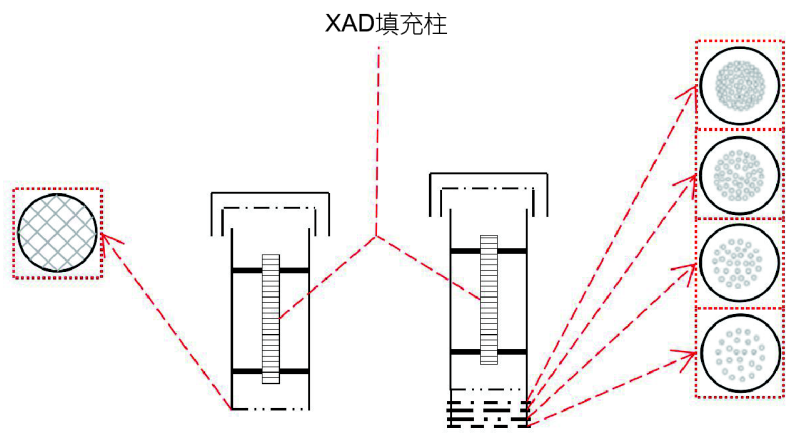

图 3 XAD-2-PAS装置示意图(左), 改进后的XAD-2-PAS装 置示意图(右) $)^{[57,67]}$ (网络版彩图)

Figure 3 The schematic drawing of original and improved XAD-2-PAS $[57,67]$ (color online).

性, 为复杂地形地区大气POPs观测提供了有力的工具. 目前PAS已广泛应用于南极、北极和青藏高原等 偏远地区大气中POPs的监测工作. XAD-2-PAS、PUFPAS、FTS等已应用于南极洲西部乔治王岛King Sejong站 ${ }^{[71]}$ 、中国长城站 ${ }^{[51,85,86]}$ 、南极洲东部罗斯海沿 岸 ${ }^{[87]}$ 以及Wilkes站 ${ }^{[84]}$ 地区附近大气中OCPs、PCBs、 PBDEs以及手性有机氯等化合物的监测工作. XAD-2PAS、PUF-PAS、SIP-PAS和FTS也在GAPS北极站点 (加拿大Alert、挪威Ny-Alesund、冰岛Storhofdi和美 国Barrow $)^{[15,54,76,88-90]}$ 以及加拿大Little Fox Lake ${ }^{[83]}$ 等 地区开展大气中 OCPs、PCBs、PBDEs 以及中性 PFCs、VMSs、新型溴代阻燃剂、OPEs等的监测研 究. 在青藏高原地区, XAD-2-PAS、PUF-PAS、FTS等 分别用于慕士塔格 $\left(38^{\circ} \mathrm{N}\right)$ 北部至珠穆朗玛峰 $\left(28^{\circ} \mathrm{N}\right)$ 南 部, 慕士塔格 $\left(75^{\circ} \mathrm{E}\right)$ 西部至察隅 $\left(97^{\circ} \mathrm{E}\right)$ 东部范围内的乡 村/偏远地区 ${ }^{[91]}$ 以及色季拉山 ${ }^{[92 \sim 94]}$ 、纳木错 ${ }^{[95]}$ 、拉 萨 ${ }^{[94]}$ 等地区大气中 OCPs、PCBs、短链氯化石蜡 $(\mathrm{SCCP})$ 的监测工作.

\section{4 偏远区域大气中POPs分布特征及污染 现状}

南极、北极和青藏高原地区(简称“三极地区”或 “三极”)是偏远区域的典型代表，因此“三极地区”大气 中POPs污染水平很大程度上反映了全球偏远区域污 染现状，本文主要对“三极地区”大气中POPs的赋存水 平及分布特征进行了总结. 


\section{1 南极地区}

目前对南极大气中的 POPs 研究主要集中在 OCPs、PCBs和PBDEs上. HCB 是南极大气中的OCPs 的主要成分, 其浓度范围在0.6 47 pg/ $\mathrm{m}^{3}$ 之间 ${ }^{[84,87,96]}$, $\mathrm{Palmer}$ 站附近大气中检出的七氯平均浓度为 $5.64 \mathrm{pg} / \mathrm{m}^{3}$, 为浓度第二高的 $\mathrm{OCPs}^{[96]}$, 而 $\alpha-\mathrm{HCH}$ 和 DDTs浓度在 ND $1.88 \mathrm{pg} / \mathrm{m}^{3}$ 和 ND $\sim 1.63 \mathrm{pg} / \mathrm{m}^{3}$ 之

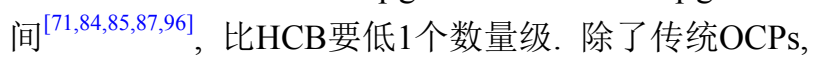
2011年被列入斯德哥尔摩公约的硫丹也备受关注, 南 极大气中以硫丹-I为主, 西南极洲King Sejong站附近 大气中测得的硫丹浓度要远高于东南极洲Wilkes站附 近大气 $\left(15.2 \mathrm{pg} / \mathrm{m}^{3} \text { vs. } 0.53 \mathrm{pg} / \mathrm{m}^{3}\right)^{[71,84]}$. 这可能是由于 西南极洲与南美洲等大陆距离较近, 与东南极洲研究 位点相比, 其他如PCBs等POPs浓度也普遍较高 ${ }^{[10,87]}$. $\mathrm{HCB}$ 和 $\mathrm{HCHs}$ 在南极大气中分布比较均匀, $\mathrm{HCB}$ 在东 南极洲罗斯海海岸 ${ }^{[87]}$ 大气中浓度平均值为 $22.9 \mathrm{pg} / \mathrm{m}^{3}$, Wilkes站附近 ${ }^{[84]}$ 为 $12.6 \mathrm{pg} / \mathrm{m}^{3}$, 西南极洲Palmer站附 近 ${ }^{[96]}$ 为 $33.8 \mathrm{pg} / \mathrm{m}^{3}$, 与 Liu等 ${ }^{[27]}$ 在卧龙自然保护区发现 $\mathrm{HCB}$ 在各个区域浓度水平类似, $\alpha-\mathrm{HCH}$ 在东南极洲 Wilkes 站 ${ }^{[84]}$ 为 $0.36 \mathrm{pg} / \mathrm{m}^{3}$, 西南极洲长城站 ${ }^{[85]}$ 为 $0.32 \mathrm{pg} / \mathrm{m}^{3}$, 这一方面可能和 $\mathrm{HCB}$ 和 $\mathrm{HCH}$ 具有较强的 挥发性及稳定性有关, 另一方面也可能与这几类污染 物退出使用时间较早, 在全球大气中分配比较平衡有 关. 东、西南极洲地区(Wilkes站和Palmer站) ${ }^{[84,96]}$, DDTs的降解产物DDEs浓度均高于DDTs, 说明南极环 境中的DDTs主要来源于长距离传输, 附近并没有新源 的输入.

环境化学与生态毒理学国家重点实验室张庆华课 题组 ${ }^{[10,51,85,86]}$ 以南极长城站为中心, 通过PUF-PAS、

XAD-2-PAS和AAS 3种采样器对南极大气中的POPs 环境行为开展了一系列持续性的研究工作. 他们将 PUF-PAS应用于南极地区大气中PCBs和PBDEs的监 测研究 ${ }^{[51]}$, 并根据前期研究得出风速与PUF-PAS的采 样速率 $(R)$ 之间存在相关关系 ${ }^{[50]}$, 推算出南极较高的风 速 $(7.3 \mathrm{~m} / \mathrm{s})$ 下PUF-PAS的 $R$ 值为 $14 \mathrm{~m}^{3} / \mathrm{d}$, 并将PAS测得 的PCBs和PBDEs浓度与 AAS数据进行了比较, 两者比 值分别在1.9 5.4和0.57 2.6之间, 证实了利用PAS采样 器研究极地地区POPs的可行性, 也进一步确认原研究 中风速与 $R$ 值之间相关关系的合理性. 由于PUF-PAS采 样周期相对较短, 他们先后采用了XAD-2-PAS和AAS
对该区域大气中POPs进行了长期监测, 通过XAD-2PAS测得的PCBs 总浓度 $\left(\sum \mathrm{PCBs}\right)$ 在 $26.7 \sim 45.1 \mathrm{pg} / \mathrm{m}^{3}$ 之 间, $\mathrm{AAS}$ 测得的大气中PCBs $\left(\sum_{20} \mathrm{PCBs}\right)$ 浓度在 $5.87 \sim 72.7 \mathrm{pg} / \mathrm{m}^{3}$ 之间, 二者具有较好的一致性, 所测得 的PCBs浓度也与Choi 等 ${ }^{[71]}$ 在韩国南极科考站附近的 研究结果较为一致. 他们的研究发现PCB-11是南极大 气中丰度最大的单体，约占PCBs总浓度的 $79 \%$, 其次 为指示性 $\mathrm{PCBs}$ ，其浓度约占 $20 \%{ }^{[10]}$, $\mathrm{PCB}$ 单体分布以 tetra-、 tri-、di-PCBs 为主, 与Khairy等 ${ }^{[96]}$ 对西南极洲 大气中PCBs的后续研究一致, 总体上, PCBs污染特征 体现了其随大气长距离传输(LRAT)的特点. 值得注意 的是, 南极大气中检测到高水平的PCB-11, 该单体并 不是商用 $\mathrm{PCBs}$ 所含单体, 而是油漆、颜料制造业的副 产品. 一方面张庆华等 ${ }^{[10]}$ 的研究发现PCB-11与PCB28、-52等传统PCBs季节变化趋势相反, 说明PCB-11 可能具有与传统 PCBs 不同的污染来源; 另一方面, Khairy等 ${ }^{[96]}$ 的研究发现南极大气中可降解为PCB-11 的单体PCB-77、-12、-169均处于未检出水平 ${ }^{[96,97]}$. 2011 年1月 2014年1月三年间, 大气中的 PCBs 和 PBDEs的水平无显著的下降趋势, 但是低氯代PCBs和 低溴代PBDEs单体在2011 2012年间呈下降趋势, 这可 能与 2012 年降雪量较大有关 ${ }^{[10]}$. 南极大气中PBDEs ( $\left.\sum_{14} \mathrm{PBDEs}\right)$ 的浓度在0.6 16.1 $\mathrm{pg} / \mathrm{m}^{3}$ 之间, 其中BDE209 丰度最大, 其次为BDE-28、-17、-47, 其他商用 penta-、octa-、deca-BDEs的主要组分如BDE-99、 $-100 、-153 、-154 、-183 、-206 、-207$ 等均有检出, 这 也是该区域大气中PBDEs赋存的首次报道 ${ }^{[51]}$. 除了对 POPs整体赋存水平的监测外, 张庆华课题组 ${ }^{[85]}$ 还对南 极大气中PCBs的手性特征进行了研究, 发现大气中手 性PCBs的对映体组成特征受到了水-气交换行为的影 响, 说明二次源排放也影响着南极大气中PCBs的分布.

Dreyer等 ${ }^{[98]}$ 于 2008 年采集南极洲附近海域大气样 品, 报道了南极大气中的PFASs的赋存情况, 该海域大 气中均能检出PFASs, 其中主要成分为PFASs的前驱体 FTOHs，6:2FTOH、8:2FTOH和 10:2FTOH总浓度为 $4 \mathrm{pg} / \mathrm{m}^{3}$, 与相近纬度大气中PCBs单体一一有机氯农药 的浓度处于同一数量级 ${ }^{[71,99]}$. Vento等 ${ }^{[100]}$ 在两年后对 南极半岛大气中PFASs进行了研究, 与Dreyer等 ${ }^{[98]}$ 的 研究一致, 南极区域大气中最主要的PFASs 是FTOHs, 其中 $8: 2$ 和 $10: 2 \mathrm{FTOH}$ 分别为 9.9 和 $7.4 \mathrm{pg} / \mathrm{m}^{3}$. Wang 等 ${ }^{[101]}$ 于2010 2011年间随德国Polarstern科考船，采集 
从北大西洋至南极菲尔德斯半岛附近的大气样品, PFASs 平均浓度为 $29.8 \mathrm{pg} / \mathrm{m}^{3}$, 主要的 PFASs 为

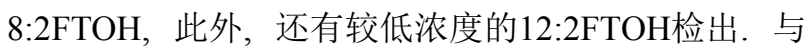
Dreyer和Vento等的研究相比, 南极大气PFASs浓度有 所上升, 值得引起注意.

马新东等 ${ }^{[102]}$ 借助我国第29次南极科考, 在南极菲 尔德斯半岛采集了大气样品，系统研究了氯化石蜡在 南极大气中的污染特征、污染来源以及气/固分配行 为. SCCPs在南极全部样品中均有检出, 乔治亚王岛大 气颗粒相和气相中 $\Sigma S C C P s$ 的浓度在9.6 20.8 pg/m $\mathrm{m}^{3}$ 之 间，平均值为 $14.9 \mathrm{pg} / \mathrm{m}^{3}$. 同族体分布，气相中以 $\mathrm{C} 10$ CPs和C11-CPs 为主, 二者之和占总量的 $56.9 \%$. 颗粒相 中则以 $12-\mathrm{CPs}$ 为主, 平均比重为 $24.1 \%$.

此外, Bengtson Nash等 ${ }^{[84]}$ 在南极检出了氟乐灵(二 硝基苯胺类除草剂), 浓度范围在 $\mathrm{ND} 13 \mathrm{fg} / \mathrm{m}^{3}$ 之间, 并 且氟乐灵在2010 2014年内呈上升趋势, 说明氟乐灵具 有长距离传输的能力, 需更加重视.

\section{2 北极地区}

北极周边被欧亚大陆及北美洲环绕，因此该区域 大气中POPs的污染水平以及环境行为是北冰洋周边 环绕国家关注的焦点，GAPS、北极监测与评估项目 (AMAP) 都在北极建有长期观测站. 北极大气中的 POPs研究种类较南极广泛，除了 OCPs、PCBs、

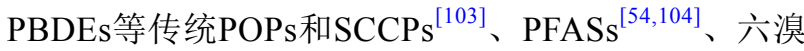

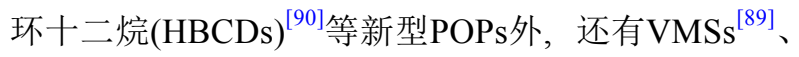
新型溴系阻燃剂 $(\mathrm{NBFR})^{[90]} 、 \mathrm{OPEs}{ }^{[90]}$ 等类POPs. GAPS北极 4 个站点(美国Barrow站、加拿大Alert站、 欧洲Ny-Alesund站以及Storhofdi站)的大气监测结果显 示 $^{[76]}$, 与南极相似, 大气中的 $\mathrm{HCB}$ 水平比较一致, 浓度 在86 98 pg/m $\mathrm{m}^{3}$ 之间，也是北极大气中OCPs的主要成 分, 比 $\alpha-\mathrm{HCH} 、 \gamma-\mathrm{HCH}$ 的浓度高4 20倍. $\gamma-\mathrm{HCH} 、 \sum$ 氯 丹 $(t r a n s$-氯丹、cis-氯丹、trans-九氯)以及 PCBs在 Storhofdi站最高, 分别为8.6、12.7、17.6 pg/m $\mathrm{m}^{3}$, Barrow站次之, Alert站和Ny-Alesund站均较低. $\gamma$ - $\mathrm{HCH}$ 在 Ny-Alesund站低于检出限, PCBs在Alert站最低, 为 $0.5 \mathrm{pg} / \mathrm{m}^{3}, \sum$ 氯丹在Alert站和Ny-Alesund站均低于检 出限. PBDEs在北极 4 个站点大气浓度在 $0.2 \sim 53 \mathrm{pg} / \mathrm{m}^{3}$ 之间, 单体分布以BDE-47、-99为主. 加拿大Alert站点 大气中PBDEs浓度较欧洲北极高，这一方面可能与北 美地区PBDEs历史用量较大有关，另一方面也可能与
欧洲限制使用PBDEs较早有关.

SCCPs虽然在2017年才纳入公约, 但其在北极的研 究历史可以追溯到1999年，Borgen等 ${ }^{[103]}$ 在挪威斯瓦尔 巴德群岛Zeppelin测得SCCPs浓度介于9.0 57 pg/ $\mathrm{m}^{3}$ 之 间. 2014年挪威环境署报告中指出Zeppelin SCCPs年度 平均值为 $240 \mathrm{pg} / \mathrm{m}^{3}$, 月均值区间为 $140 \sim 480 \mathrm{pg} / \mathrm{m}^{3[105]}$, 远高于同时期PCBs等传统POPs ${ }^{[83]}$ ，与1999年的结果 相比, 有一定的增长.

北极大气中也检测到了硫丹类化合物，分布特征 与南极相似，以硫丹-I为主，2009年GAPS数据显示北 极站点大气中硫丹-I的浓度在7.8 19 $\mathrm{pg} / \mathrm{m}^{3}$ 之间 ${ }^{[76]}$.

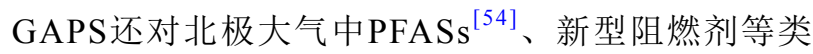
POPs进行了研究, 大气中主要的PFASs 是 $8: 2 \mathrm{FTOH}$, 浓 度在6.4 7.6 pg/m $\mathrm{m}^{3}$ 之间, PFOS浓度在BDL $2.0 \mathrm{pg} / \mathrm{m}^{3}$ 之 间, Alert站浓度较高, 为 $2.0 \mathrm{pg} / \mathrm{m}^{3}$; 新型阻燃剂六溴苯 (HBB)、德克隆602 (Dechlorane 602)以及2-乙基己基2,3,4,5-四溴苯甲酸(EH-TBB)检出率较高, 浓度分别在 $<0.06 \sim 0.6 \mathrm{pg} / \mathrm{m}^{3} 、<0.01 \sim 0.78 \mathrm{pg} / \mathrm{m}^{3}$ 以及 $<0.8 \sim$ $529 \mathrm{pg} / \mathrm{m}^{3}$ 之间, HBCDs在 4 个北极站点大气中的浓度 均低于检出限 ${ }^{[90]}$. 北极大气中磷酸三 (2-氯乙基)酯 (TCEP)、磷酸三(氯异丙基)酯(TCPP)、磷酸三苯酯 (TPP)、磷酸三(2-丁氧基乙基)酯(TBEP)检出频率较 高, 4 个站点 $\sum O P E s$ 分别在 $87 \sim 1358 、 783 \sim 1722$ 、 120 2500以及20 511 pg/m $\mathrm{m}^{3}$ 之间 ${ }^{[90]}$ ，浓度高于传统 POPs，值得引起重视．此外，环状挥发性甲基硅氧烷 (cVMS) 也在 4 个站点大气检出 ${ }^{[89]}$, 浓度均值分别达到 $1.6 、 22.9 、 37.5 、 2.2 \mathrm{pg} / \mathrm{m}^{3}$ ，几乎与 $\alpha-\mathrm{HCH}$ 、硫丹-I等 传统POPs相当.

AMAP中对北极大气进行了长达20年(1993 2012 年)的监测 ${ }^{[106]}$, 北极大气中PCBs、DDTs以及氯丹等大 部分POPs均呈缓慢下降趋势. 对比2005和2009年的 GAPS数据，部分污染物在 4 个站点间趋势并不完全相 同 ${ }^{[15,76]}$, 硫丹-I、PCBs在 4 个站点均呈下降趋势, Alert 站大气中的PCBs由 2005 年的 $27.5 \mathrm{pg} / \mathrm{m}^{3}$ 下降至2009年 的 $0.5 \mathrm{pg} / \mathrm{m}^{3} ; \gamma-\mathrm{HCH}$ 在Alert站、Ny-Alesund站以及 Storhofdi站均有下降，其中Ny-Alesund站由 $5 \mathrm{pg} / \mathrm{m}^{3}$ 降 至未检出水平; $\sum$ 氯丹在Alert站、Ny-Alesund站均由 $4 \mathrm{pg} / \mathrm{m}^{3}$ 降至未检出水平，但Storhofdi站略有上升. 这 些研究说明公约管控对POPs削减具有一定的成效, 但 POPs在大气中的浓度还受到很多其他因素的影响. 近 年来全球气候变暖导致北极冰川融化，冰川中俘获的 
一些污染物会再次挥发到大气中, HCB在Storhofdi、 Zeppelin大气中浓度近年来呈上升趋势, 低氯代PCBs 单体(PCB-52、-101)在Storhofdi大气中呈现增长趋势, 可能受到了冰盖融化而释放的POPs二次源影响.

此外，一些现用杀虫剂杜克、五氯硝基苯、氟乐 灵等均在北极大气中检出 ${ }^{[106]}$, 说明现在这些大量使用 的化学品也具有一定的长距离传输能力, 需加强对这 些化学品的进一步评估.

\section{3 青藏高原地区}

青藏高原(Tibetan Plateau, TP)平均海拔高达 $4000 \mathrm{~m}$, 有“世界第三极”之称, 其腹地年均温度在 $0^{\circ} \mathrm{C}$ 以下, 是世界上最冷的地区之一. 由于当地稀疏的人口 密度以及几乎不存在的工业生产活动，除发现拉萨市 存在OCPs当地污染源外 ${ }^{[107]}$, 未见其他POPs当地源的 报道. 相对于北美与欧洲偏远山区，青藏高原地区 POPs较系统的研究开始较晚, 但近年来研究者对该地 区的关注程度日益增大. 越来越多的研究表明，TP没 有对现代工农业产生的污染独善其身，很多《斯德哥 尔摩公约》所涉及的POPs在青藏高原生态系统中均 有发现 ${ }^{[93 ~ 95,108]}$. TP大气中的POPs 研究包括OCPs、 PCBs、PBDEs等传统POPs ${ }^{[92,93,95,109]} 、 \mathrm{SCCPs}^{[94]}$ 、 $\mathrm{HBCDs}^{[93,108]}$ 等新型POPs以及PAHs ${ }^{[95,110]}$ 、非PBDEs 阻燃剂 ${ }^{[108,111]}$ 等类POPs. TP大气中丰度较大的OCPs 为 $\mathrm{HCHs}$ 、DDTs和 $\mathrm{HCB}$, 其中 $\alpha-\mathrm{HCH}$ 和 $\gamma-\mathrm{HCH}$ 分别在 $0.1 \sim 144$ 和ND $24.3 \mathrm{pg} / \mathrm{m}^{3}$ 之间, DDTs在0.4 540 pg/m $\mathrm{m}^{3}$ 之间, $\mathrm{HCB}$ 在ND $85 \mathrm{pg} / \mathrm{m}^{3}$ 之间 ${ }^{[92,93,95,109,110,112]}$; 浓度指 示型PCBs单体(包括PCB-28、-52、-101、-138、-153 以及-180)浓度和在 ND $30.1 \mathrm{pg} / \mathrm{m}^{3}$ 之间, 单体分布以 PCB-28、-52为主 ${ }^{[91,93]}$; PBDEs 在纳木错、贡嘎山东坡 及色季拉山大气中浓度在分别为 $0.8 \sim 5.2 、 0.4 \sim 8.7$ 、 $\mathrm{ND} \sim 0.26 \mathrm{pg} / \mathrm{m}^{3}$ 之间, 单体分布分别以BDE-47、-99、100, BDE-28、-47, BDE-47、-28、-183 为主 ${ }^{[95,111,113]}$; 拉萨地区大气中PBDEs浓度中值高达 $40 \mathrm{pg} / \mathrm{m}^{3}$, 以 deca-、octa-、nona-BDEs单体为主, 该地区大气中 PBDEs水平及分布特征与其他TP地区差异较大, 一方 面可能因为拉萨市人口密度较大, 当地具有商用decaBDEs的污染源, 另一方面可能与该研究中PBDEs浓度 是大气相和颗粒相的总和, 大气颗粒相中富集了大量 高溴代PBDEs ${ }^{[108]}$. 青藏高原硫丹类化合物以硫丹-I为 主, 且在纳木错、色季拉山地区大气水平相似, 浓度
均值分别为 75.4 和 $78 \mathrm{pg} / \mathrm{m}^{3[93,95]}$, 比南极和北极地区 高 $^{\left[{ }^{[76,84]}\right.}$.

中国科学院青藏高原研究所王小萍团队 ${ }^{[91]}$ 利用 $\mathrm{XAD}$ 被动采样器在青藏高原建立了大气被动采样网 络, 对TP地区大范围内大气中POPs分布进行了长期的 监测. 她们发现 $\mathrm{HCHs}$ 的最高值出现在中印边境附近的 高海拔 $(>4000 \mathrm{~m}$ ) 采样点上, 这说明青藏高原大气 $\mathrm{HCHs}$ 的空间分布可能受到南亚污染物长距离大气传 输的影响; HCB、PCBs、PBDEs和PAHs的最高浓度 都出现在波密, 有可能是该采样点附近频繁的森林火 灾导致这些化合物的排放或二次源排放 ${ }^{[73]}$. 总体上, $\mathrm{TP}$ 大气中的POPs主要来自印度季风或西风驱动下中 亚或欧洲等地区POPs的长距离传输作用, TP地区东南 部、北部/西部、中部大气中POPs分布各具特点, 其中 东南部大气中DDTs的浓度及其在大气POPs中所占百 分比(占比)较高, 该区域主要为印度季风气候控制, 而 DDTs、HCHs 是印度大气中主要的POPs, 因此推测TP 东南部大气中的POPs主要来自印度地区POPs的长距 离传输作用; TP北部/西部大气中HCB占比较大, 该区 域主要受西风系统影响, 气团来自欧洲、中亚, 欧洲地 区大气中POPs水平整体较低且以 $\mathrm{HCB}$ 为主, 因此推测 TP西部大气中POPs主要受欧洲地区POPs的LRAT影 响; TP中部, HCB、DDTs占比均较大, 受印度季风与 西风共同影响, 因此大气中POPs分布表现为印度与欧 洲地区POPs的长距离传输作用共同影响. 印度季风、 西风分别在夏季和冬季盛行, 因此TP地区大气中大部 分POPs 具有夏天高、冬天低的明显的季节性变化. 在 5 年的大气监测研究中 ${ }^{[91]}, \mathrm{HCHs} 、 \mathrm{HCB}$ 及 $\mathrm{PCBs}$ 均呈下 降趋势, 但TP东南部大气中的 $o, p^{\prime}$-DDT、 $p, p^{\prime}$-DDT没 有明显的下降趋势, 该区域是印度POPs排放受体区, 印度地区对DDTs的管控仍有待加强.

近年来, 环境化学与生态毒理学国家重点实验室 江桂斌课题组 ${ }^{[93,94]}$ 利用XAD-2-PAS对藏东南色季拉山 地区大气中 OCPs、PCBs、PBDEs等传统POPs 和 HBCDs、SCCPs等新型POPs浓度水平及其海拔梯度 变化趋势进行了研究, 并探究了温度、降水、季风和 植被等不同环境因素的影响. 与已有研究比较, 色季 拉山大气中 $\alpha-\mathrm{HCH} 、 \gamma-\mathrm{HCH} 、 \mathrm{DDTs}$ 与 TP地区已有研 究结果较为一致 ${ }^{[92,109,110,112]}$, 硫丹-I和PCBs均与纳木错 地区水平相似 ${ }^{[95]}$; 大气中的PBDEs浓度水平较TP其他 地区低 ${ }^{[108,111,113]}$. 他们根据大气中POPs的污染特征, 推 


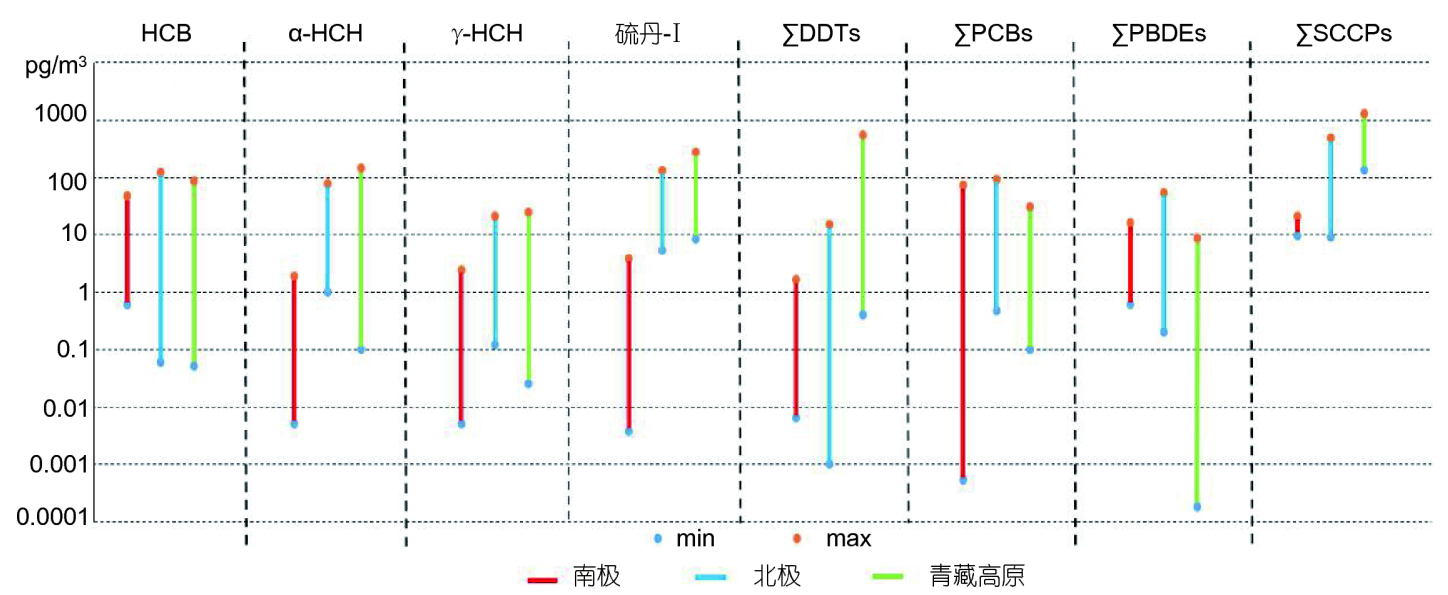

图 4 南极、北极、青藏高原地区大气主要POPs浓度水平对比(具体数据及参考文献详见补充资料) (网络版彩图) Figure4 The levels of atmospheric POPs in Antarctic, Arctic and Tibetan Plateau (color online).

测该区域DDTs、HCHs以及硫丹的主要来源均为其他 源区POPs的LRAT作用，其中DDTs、HCHs分别同时 受商用DDTs和三氯杀螨醇、商用HCHs和林丹共同影 响. 色季拉山东坡大气样品中的POPs浓度水平和海拔 梯度之间不存在显著相关性，可能与雅鲁藏布江流域 水汽沿东坡输送导致东坡大气局部运动较强有关. 西 坡大气样品中DDTs及硫丹的浓度水平随海拔的升高 而增加，呈现显著正相关性，揭示DDTs和硫丹在西藏 色季拉山地区存在潜在的局部高山冷凝效应. 色季拉 山大气中 HBCDs浓度较PBDEs 高, 浓度在 ND $2.84 \mathrm{pg} / \mathrm{m}^{3}$ 之间, 平均浓度为 $0.25 \mathrm{pg} / \mathrm{m}^{3}$, 异构体组成与 商用HBCDs一致. SCCPs浓度在132 1267 pg/m²间, 平均浓度为 $457.5 \mathrm{pg} / \mathrm{m}^{3}$ ，同族体分布以C10-、C11-CPs 以及C16-、C17-CPs为主．色季拉山大气中HBCDs和 SCCPs都较拉萨低 1 个数量级. 此外, 分析结果显示, 森林内大气样品中POPs浓度低于周边空地大气样品中 的POPs浓度, 部分POPs森林滤过率可达 $50 \%$, 在一定程 度上证明了藏东南地区森林过滤效应的存在 ${ }^{[93,94]}$.

此外, 已有研究在TP地区大气中检出了DBDPE、 TBPH、EH-TBB等新型阻燃剂, 浓度分别在 $23 \sim 41.3$ 、 0.8 0.94以及 $0.71 \sim 0.74 \mathrm{pg} / \mathrm{m}^{3}$ 之间 ${ }^{[108,111,113]}$, 氟乐灵等 杀虫剂在纳木错大气中也有检出 ${ }^{[95]}$.

\section{5 结论与展望}

被动式大气采样器被广泛地应用于南极、北极和
青藏高原等偏远区域，成功地表征了当地大气POPs污 染水平, 为传统POPs、新列入公约的POPs以及新型溴 系和磷系阻燃剂在极地大气中赋存和环境行为的研究 提供了平台, 同时还有助于评估公约履约进程和POPs 对当地生态系统构成的潜在威胁.

与主动式大气采样器相比, 不同环境气候条件下 被动采样器的 $R$ 值波动对采样体积影响较大, 但这方 面的研究相对较少. 王小萍等 ${ }^{[67]}$ 的研究表明大气被动 采样器外罩的设计有助于抵消外部环境干扰造成的 $R$ 值变化, 这种 $R$ 值稳定的改进型XAD-2-PAS在极地等 气象条件恶劣区域的应用将有助于全球大气POPs观 测结果的横向比较, 但总体上XAD-2-PAS的采样效率 偏低, 后续研究有必要继续开发在不同气象条件下, $R$ 值稳定且采样速率较高的新型采样器. 此外, 部分 POPs替代品在物化性质上均与原有产品类似, 偏远区 域大气监测除公约关注的POPs外, 不断有新型POPs的 检出, 在今后的研究中, 对于具有类POPs性质的污染 物需纳入监测范围中, 以最大程度地避免或减轻其对 环境及生态可能造成的危害. 随着监测名单的不断增 长, 现有采样介质有可能存在不能全面采集各类大气 污染物的情况, 因此, 现有采样介质在研究新型污染 物时需要及时对吸附性能进行评估, 并且随着材料科 学的进展, 不断地筱选适合大气采样的优良吸附介质.

“三极”由于所处地理环境存在较大的不同，POPs 在“三极”的分布也各有特点(图4, 详细信息见网络版 补充材料), 南极由于远离工农业密集分布的大陆, 周 
边基本没有污染源的排放, 污染物通过长距离传输进 入, 大气中主要以 $\mathrm{HCB}$ 等禁用较长时间的POPs为主. 北极大气中HCB等禁用时间较长的OCPs占比较高, 但 北极被一系列发达国家所包围, 在传统POPs被限制生 产使用后, 很多POPs的新型替代品在北极大气中检出 率较高, 如OPEs等新型污染物在北极大气中浓度达到 $了 10^{2} \sim 10^{3} \mathrm{pg} / \mathrm{m}^{3}$. 青藏高原处于一系列发展中国家的 包围, 各个国家工农业生产粗放, 对环境的关注度较 低, 来自南亚的印度季风是青藏高原POPs的重要来 源, 青藏高原大气中DDTs、硫丹等农药含量要高于南 极和北极. 值得注意的是, 不少研究发现拉萨市大气中 POPs浓度要显著高于青藏高原其他区域. 青藏高原作
为我国西南部生态屏障, 除了关注公约内POPs跨境传 输外, 青藏高原还面临新型POPs的挑战.

在后公约时代, POPs排放的一次源受到限制, 偏 远区域的POPs污染趋势备受关注，极地大气中不同 POPs的变化趋势并不一致. 部分较早停止生产使用的 POPs, 如HCHs和PCBs, 在这些区域大气中均呈一定的 下降趋势; 但部分POPs如DDTs, 由于其在一些应用领 域的替代品效果不佳, 或具有豁免权, 因此环境中仍存 在其一次源, 这种污染特征也体现在青藏高原大气环 境中. 此外, 近年来气候变暖导致极地冰川融化, 冷凝/ 俘获在冰川的POPs成为二次源, 重新进入环境, 值得 引起关注.

\section{致谢感谢国家海洋局极地考察办公室极地研究中心和中国科学院青年科学促进会对南北极现场科考的大力支持.}

\section{补充材料}

本文的补充材料见网络版http://chemen.scichina.com. 补充材料为作者提供的原始数据, 作者对其学术质量和内容负责.

\section{参考文献}

1 Sladen WJL, Menzie CM, Reichel WL. Nature, 1966, 210: 670-673

2 Qiu J. Nature, 2013

3 Galbán-Malagón C, Berrojalbiz N, Ojeda MJ, Dachs J. Nat Commun, 2012, 3: 862

4 Jamieson AJ, Malkocs T, Piertney SB, Fujii T, Zhang Z. Nat Ecol Evol, 2017, 1: 0051

5 Kelly BC, Ikonomou MG, Blair JD, Morin AE, Gobas FAPC. Science, 2007, 317: 236-239

6 Ren A, Qiu X, Jin L, Ma J, Li Z, Zhang L, Zhu H, Finnell RH, Zhu T. Proc Natl Acad Sci USA, 2011, 108: 12770-12775

7 Köhler HR, Triebskorn R. Science, 2013, 341: 759-765

$8 \mathrm{http}: / / \mathrm{chm}$. pops.int/TheConvention/ThePOPs/AllPOPs/tabid/2509/Default.aspx (Accessed in April, 2, 2018)

9 UNEP/POPS/COP.4/33. Global monitoring report under the global monitoring plan for effectiveness evaluation. UNEP, 2009

10 Wang P, Li Y, Zhang Q, Yang Q, Zhang L, Liu F, Fu J, Meng W, Wang D, Sun H, Zheng S, Hao Y, Liang Y, Jiang G. Atmos Environ, 2017, 150: $407-416$

11 Muñoz-Arnanz J, Roscales JL, Ros M, Vicente A, Jiménez B. Environ Pollut, 2016, 217: 107-113

Chaemfa C, Barber JL, Huber S, Breivik K, Jones KC. J Environ Monit, 2010, 12: 1100-1109

Melymuk L, Bohlin P, Sáňka O, Pozo K, Klánová J. Environ Sci Technol, 2014, 48: 14077-14091

Gouin T, Harner T, Blanchard P, Mackay D. Environ Sci Technol, 2005, 39: 9115-9122

Pozo K, Harner T, Lee SC, Wania F, Muir DCG, Jones KC. Environ Sci Technol, 2009, 43: 796-803

Halse AK, Schlabach M, Eckhardt S, Sweetman A, Jones KC, Breivik K. Atmos Chem Phys, 2011, 11: 1549-1564

Wang XP, Gong P, Yao TD. Environ Chem, 2008, 29: 273-282 (in Chinese) [王小萍, 龚平, 姚檀栋. 环境科学, 2008, 29: 273-282]

Simonich SL, Hites RA. Environ Sci Technol, 1995, 29: 2905-2914

Wegener JWM, van Schaik MJM, Aiking H. Environ Pollut, 1992, 76: 15-18

Harmens H, Foan L, Simon V, Mills G. Environ Pollut, 2013, 173: 245-254

Shoeib M, Harner T. Environ Sci Technol, 2002, 36: 4142-4151

Van Drooge BL, Grimalt JO, Booij K, Camarero L, Catalan J. Atmos Environ, 2005, 39: 5195-5204 
Pozo K, Harner T, Lee SC, Sinha RK, Sengupta B, Loewen M, Geethalakshmi V, Kannan K, Volpi V. Environ Pollut, 2011, 159: 646-653

Bidleman TF, Laudon H, Nygren O, Svanberg S, Tysklind M. Environ Pollut, 2017, 225: 381-389

Birgül A1, Kurt-Karakus PB, Alegria H, Gungormus E, Celik H, Cicek T, Güven EC. Chemosphere, 2017, 168: 1345-1355

Liu R, Lin Y, Liu R, Hu F, Ruan T, Jiang G. Talanta, 2016, 147: 69-75

Liu W, Chen D, Liu X, Zheng X, Yang W, Westgate JN, Wania F. Environ Sci Technol, 2010, 44: 1559-1565

Shunthirasingham C, Barra R, Mendoza G, Montory M, Oyiliagu CE, Lei YD, Wania F. Atmos Environ, 2011, 45: 303-309

Petty JD, Orazio CE, Huckins JN, Gale RW, Lebo JA, Meadows JC, Echols KR, Cranor WL. J Chromatogr A, 2000, 879: 83-95

Esteve-Turrillas FA, Pastor A, Yusà V, de la Guardia M. TrAC Trends Anal Chem, 2007, 26: 703-712

Qu CK, Doherty AL, Xing XL, Sun W, Albanese S, Lima A, Qi SH, Vivo BD. Polyurethane Foam-Based Passive Air Samplers in Monitoring Persistent Organic Pollutants: Theory and Application. 2ed. Environmental Geochemistry, 2018

Zhu Q, Liu G, Zhang X, Dong S, Gao L, Zheng M. Asian J Ecotoxicol, 2016, 11: 50 -60 (in Chinese) [朱青青, 刘国瑞, 张宪, 董姝君, 高丽荣, 郑明辉. 生态毒理学报, 2016, 11: 50-60]

Zhang G, Liu X. Prog Chem, 2009, 21: 297-306 (in Chinese) [张干, 刘向. 化学进展, 2009, 21: 297-306]

Soedergren A. Environ Sci Technol, 1987, 21: 855-859

Huckins JN, Tubergen MW, Manuweera GK. Chemosphere, 1990, 20: 533-552

Petty JD, Huckins JN, Zajicek JL. Chemosphere, 1993, 27: 1609-1624

Ockenden WA, Prest HF, Thomas GO, Sweetman A, Jones KC. Environ Sci Technol, 1998, 32: 1538-1543

Ockenden WA, Sweetman AJ, Prest HF, Steinnes E, Jones KC. Environ Sci Technol, 1998, 32: 2795-2803

Fiedler S, Pfister G, Schramm KW. Environ Sci Pollut Res, 2010, 17: 420-428

dos Reis LGT, Gallart-Mateu D, Pacheco WF, Pastor A, de la Guardia M, Cassella RJ. Microchem J, 2013, 110: 494-500

Bowen HJM. J Chem Soc A, 1970, 1082

Gesser HD, Chow A, Davis FC, Uthe JF, Reinke J. Anal Lett, 1971, 4: 883-886

Bidleman TF, Olney CE. Bull Environ Contam Toxicol, 1974, 11: 442-450

Jaward FM, Farrar NJ, Harner T, Sweetman AJ, Jones KC. Environ Toxicol Chem, 2004, 23: 1355-1364

Jaward FM, Farrar NJ, Harner T, Sweetman AJ, Jones KC. Environ Sci Technol, 2004, 38: 34-41

Pozo K, Harner T, Shoeib M, Urrutia R, Barra R, Parra O, Focardi S. Environ Sci Technol, 2004, 38: 6529-6537

Harner T, Shoeib M, Diamond M, Stern G, Rosenberg B. Environ Sci Technol, 2004, 38: 4474-4483

Wang C, Wang X, Ren J, Gong P, Yao T. Sci Total Environ, 2017, 580: 958-965

Tuduri L, Harner T, Hung H. Environ Pollut, 2006, 144: 377-383

Li Y, Zhang Q, Ji D, Wang T, Wang Y, Wang P, Ding L, Jiang G. Environ Sci Technol, 2009, 43: 1030-1035

Li Y, Geng D, Liu F, Wang T, Wang P, Zhang Q, Jiang G. Atmos Environ, 2012, 51: 140-145

Chaemfa C, Barber JL, Gocht T, Harner T, Holoubek I, Klanova J, Jones KC. Environ Pollut, 2008, 156: 1290-1297

Hayward SJ, Gouin T, Wania F. Environ Sci Technol, 2010, 44: 3410-3416

Genualdi S, Lee SC, Shoeib M, Gawor A, Ahrens L, Harner T. Environ Sci Technol, 2010, 44: 5534-5539

Gawor A, Shunthirasingham C, Hayward SJ, Lei YD, Gouin T, Mmereki BT, Masamba W, Ruepert C, Castillo LE, Shoeib M, Lee SC, Harner T, Wania F. Environ Sci-Proc Impacts, 2014, 16: 404-413

UNEP. Guidance for a Global Monitoring Programme for Persistent Organic Pollutants. 2004

Wania F, Shen L, Lei YD, Teixeira C, Muir DCG. Environ Sci Technol, 2003, 37: 1352-1359

Shen L, Lei YD, Wania F. J Chem Eng Data, 2002, 47: 944-949

Hayward SJ, Lei YD, Wania F. Atmos Environ, 2011, 45: 296-302

Gouin T, Wania F, Ruepert C, Castillo LE. Environ Sci Technol, 2008, 42: 6625-6630

Zhang X, Tsurukawa M, Nakano T, Lei YD, Wania F. Environ Sci Technol, 2011, 45: 10509-10515

Zhang X, Wania F. Environ Sci Technol, 2012, 46: 9563-9570

Armitage JM, Hayward SJ, Wania F. Environ Sci Technol, 2013, 47: 13546-13554

Zhang X, Brown TN, Ansari A, Yeun B, Kitaoka K, Kondo A, Lei YD, Wania F. Environ Sci Technol, 2013, 47: 7868-7875

Zhang X, Hoang M, Lei YD, Wania F. Environ Sci-Proc Impacts, 2015, 17: 2006-2012 
Gong P, Wang X, Liu X, Wania F. Environ Sci Technol, 2017, 51: 5642-5649

Zhang XM. Passive air samplers for semivolatile organic compounds: experiments, modeling, and field application. Dissertation for the Doctoral Degree. Toronto: University of Toronto, 2012

69 Shen L, Wania F, Lei YD, Teixeira C, Muir DCG, Bidleman TF. Environ Sci Technol, 2005, 39: 409-420

70 Shen L, Wania F, Lei YD, Teixeira C, Muir DCG, Xiao H. Environ Pollut, 2006, 144: 434-444

Choi SD, Baek SY, Chang YS, Wania F, Ikonomou MG, Yoon YJ, Park BK, Hong S. Environ Sci Technol, 2008, 42: 7125-7131

Daly GL, Lei YD, Teixeira C, Muir DCG, Castillo LE, Jantunen LMM, Wania F. Environ Sci Technol, 2007, 41: 1124-1130

Liu X, Zheng X, Wang B, Jiang G. Passive Atmospheric Sampling Technique of POPs and Its Regional Atmospheric Transportation. Beijing: Science Press, 2015 (in Chinese) [刘咸德, 郑晓燕, 王宝盛, 江桂斌. 持久性有机污染物被动采样与区域大气传输. 北京: 科学出版社, 2015] Shoeib M, Harner T, Lee SC, Lane D, Zhu J. Anal Chem, 2008, 80: 675-682

Koblizkova M, Genualdi S, Lee SC, Harner T. Environ Sci Technol, 2012, 46: 391-396

Wang P, Zhang Q, Li Y, Zhu C, Chen Z, Zheng S, Sun H, Liang Y, Jiang G. Sci Rep, 2015, 5: 13913

Li YM, Geng DW, Hu YB, Wang P, Zhang QH, Jiang GB. Chin Sci Bull, 2012, 57: 1499-1503

Pozo K, Martellini T, Corsolini S, Harner T, Estellano V, Kukučka P, Mulder MD, Lammel G, Cincinelli A. Chemosphere, 2017, 178: 458-465

Baek SY, Choi SD, Chang YS. Environ Sci Technol, 2011, 45: 4475-4482

Genualdi S, Harner T, Cheng Y, Macleod M, Hansen KM, van Egmond R, Shoeib M, Lee SC. Environ Sci Technol, 2011, 45: 3349-3354 Rauert C, Schuster JK, Eng A, Harner T. Environ Sci Technol, 2018, 52: 2777-2789

Wang X, Ren J, Gong P, Wang C, Xue Y, Yao T, Lohmann R. Atmos Chem Phys, 2016, 16: 6901-6911

Ren J, Wang X, Xue Y, Gong P, Joswiak DR, Xu B, Yao T. Environ Pollut, 2014, 194: 210-216

Zhu N, Schramm KW, Wang T, Henkelmann B, Zheng X, Fu J, Gao Y, Wang Y, Jiang G. Environ Pollut, 2014, 191: 166-174

Wu J, Gao W, Liang Y, Fu J, Gao Y, Wang Y, Jiang G. Environ Sci Technol, 2017, 51: 11136-11144

Xiao H, Kang S, Zhang Q, Han W, Loewen M, Wong F, Hung H, Lei YD, Wania F. J Geophys Res, 2010, 115: D16310

Khairy MA, Luek JL, Dickhut R, Lohmann R. Environ Pollut, 2016, 216: 304-313

Vecchiato M, Argiriadis E, Zambon S, Barbante C, Toscano G, Gambaro A, Piazza R. Microchem J, 2015, 119: 75-82

Dreyer A, Weinberg I, Temme C, Ebinghaus R. Environ Sci Technol, 2009, 43: 6507-6514

Montone RC, Taniguchi S, Boian C, Weber RR. Mar Pollut Bull, 2005, 50: 778-782

Vento SD, Halsall C, Gioia R, Jones K, Dachs J. Atmos Pollut Res, 2012, 3: 450-455

Wang Z, Xie Z, Mi W, Möller A, Wolschke H, Ebinghaus R. Environ Sci Technol, 2015, 49: 7770-7775

Ma X, Zhang H, Zhou H, Na G, Wang Z, Chen C, Chen J, Chen J. Atmos Environ, 2014, 90: 10-15

Borgen AR, Schlabach M, Gundersen H. Organohalogen Compd, 2000, 47: 272-274

Stock NL, Furdui VI, Muir DCG, Mabury SA. Environ Sci Technol, 2007, 41: 3529-3536

5 Norwegian Environmental Agency. Monitoring of Environmental Contaminants in Air and Precipitation. Norwegian Institute for Air Research M-368. 2015 Annual Report, 2014-Norwegian Institute for Air Research, 2015

106 Hung H, Katsoyiannis AA, Brorström-Lundén E, Olafsdottir K, Aas W, Breivik K, Bohlin-Nizzetto P, Sigurdsson A, Hakola H, Bossi R, Skov H, Sverko E, Barresi E, Fellin P, Wilson S. Environ Pollut, 2016, 217: 52-61 


\title{
Progress on the passive air sampling techniques and occurrence of persistent organic pollutants in remote areas
}

\author{
Jingxing Zhang ${ }^{1,2}$, Duo $\mathrm{Bu}^{3}$, Pu Wang ${ }^{1}$, Jianjie Fu ${ }^{1 *}$, Yong Liang ${ }^{2}$, Qinghua Zhang ${ }^{1}$, Aiqian Zhang ${ }^{1,2^{*}}$ \\ ${ }^{1}$ State Key Laboratory of Environmental Chemistry and Ecotoxicology, Research Center for Eco-Environmental Sciences, Chinese Academy of \\ Sciences, Beijing 100085, China \\ ${ }^{2}$ Institute of Environment and Health, Jianghan University, Wuhan 430056, China \\ ${ }^{3}$ School of Science, Tibet University, Lhasa 850000, China \\ *Corresponding authors (email: jjfu@rcees.ac.cn; aqzhang@rcees.ac.cn)
}

\begin{abstract}
Persistent organic pollutants can undergo long-range transport and deposit in remote areas (e.g., high mountains, polar areas) through "global distillation effects" or "cold condensation". This behavior of POPs would pose great risks to the fragile polar ecosystem. Passive air samplers are widely applied to air sampling program at large scales and they are very useful to monitor the pollution levels of legacy POPs in remote areas. They have been widely used to trace the environmental behavior of emerging POPs in the past few decades. The application of PAS has contributed a lot to offer fundamental data to the Stockholm Convention. This study introduced five passive air sampling techniques (SPMD-PAS, PUF-PAS, XAD-2-PAS, SIP-PAS and FTS) and their development history, especially emphasizing on their application and the current pollution of legacy and emerging POPs in remote areas, such as Antarctic, Arctic and Tibetan Plateau. Furthermore, the potential need and future application of PAS were also discussed.
\end{abstract}

Keywords: persistent organic pollutants (POPs), passive air sampling, Antarctic, Arctic, Tibetan Plateau

doi: $10.1360 / \mathrm{N} 032018-00089$ 